\title{
La Presencia del Investigador en el Ecosistema Digital de la Ciencia
}

\section{Abierta \\ The Researcher's Presence in the Open Science Digital Ecosystem}

\author{
Francisco José García-Peñalvo ${ }^{1}$, Ángel Fidalgo-Blanco ${ }^{2}$, María Luisa Sein-Echaluce ${ }^{3}$, Fernando Tricas García ${ }^{2}$ \\ fgarcia@usal.es, angel.fidalgo@upm.es,mlsein@unizar.es, ftricas@unizar.es
}

${ }^{1}$ Departamento de Informática y Automática
Universidad de Salamanca
Salamanca, España

${ }^{3}$ Departamento de Matemática Aplicada Universidad de Zaragoza

Zaragoza, España

\author{
${ }^{2}$ Departamento de Ingeniería Geológica y Minera \\ Universidad Politécnica de Madrid \\ Madrid, España
}

${ }^{4}$ Departamento de Lenguajes y Sistemas
Informáticos Universidad de Zaragoza
Zaragoza, España

de datos de referencia internacional; esto es, de Journal Citation Report (JCR), Web of Science (WoS) y Scopus. En menor medida, se tienen en consideración otros índices como Emerging Sources Citation Index (ESCI) de WoS, el Sello de Calidad de la FECYT, Scielo o el Scholarly Publishers Indicators in Humanities and Social Sciences (SPI) entre otros.

Estos índices se basan en una organización en cuartiles de las fuentes (revistas en el caso de JCR y Scopus; editoriales en el caso de SPI) o en la inclusión de la revista en la base de datos como sucede en ESCI, Scielo o el Sello de Calidad de la FECYT.

Este sistema de evaluación provoca que se evalúe a los autores no por la calidad del contenido, sino más bien por la calidad del continente. Esto se debe a que el sistema supone que las fuentes calificadas ya cuidan de la calidad sus artículos aceptados y que esta calidad influirá en la posición futura de las revistas en los cuartiles en función de las citas recibidas en la ventana de años definida.

El sistema actual cuenta con muchos detractores pero, hasta que oficialmente no se sustituya o se mejore con un sistema de indicadores multifuente, es el que deben tomar como referencia los investigadores. Con el pueden obtener el conjunto de indicadores clave con los que deben cumplimentar su currículo de cara a convocatorias competitivas, promociones, acreditaciones, etc.

Dichos indicadores se obtienen de los sistemas de información que sustentan las bases de datos de referencia, en las que el investigador debe tener un perfil que lo identifique de forma inequívoca para asignarle la producción científica sobre la que se calculan los mencionados indicadores.

Además, estos perfiles, junto con otros componentes de tipo social, contribuyen a dar visibilidad a la producción del investigador y aportar transparencia de sus contribuciones a la sociedad en general, lo cual es una de las contribuciones de la denominada Ciencia Abierta (Crue Universidades Españolas, 2019; Fecher \& Friesike, 2014; Vicente-Saez \& MartínezFuentes, 2018).

El conjunto de perfiles de investigador en estos sistemas, que conforman el ecosistema digital de la ciencia y de la 
investigación, constituyen la identidad digital del investigador, necesaria para su evaluación actual como investigador, como para cualquier variante que pudiera surgir en un futuro más o menos cercano. Además, lo que no es más que otra forma de evaluación, esta identidad digital supone la rendición de cuentas y la transparencia para la sociedad en general que, en una gran medida, es la fuente de financiación principal de las investigaciones realizadas con fondos públicos.

Por otra parte, en un sentido de corresponsabilidad y transitividad, los perfiles e indicadores de los investigadores individuales servirán como base para construir el perfil de investigación de las comunidades en las que estos estén inmersos, es decir, grupo de investigación, instituto de investigación, universidad, etc. Es decir, ya no solo es importante cuidar los perfiles digitales individuales por lo que aportan a la identidad digital y, por tanto, al desarrollo curricular de cada investigador, sino que dichos perfiles influyen en la conformación de los perfiles, y con ello en la identidad digital global, de la institución a la que se pertenece. Estos datos aportan elementos cuantitativos que redundan en los diferentes rankings institucionales y elementos cualitativos que se ven reflejados en las reputaciones científicas individuales de los investigadores y colectivas de sus instituciones.

En este artículo se va a presentar la última versión de un protocolo (García-Peñalvo, 2017a, 2018a, 2018b) para que un investigador pueda mantener perfiles digitales en los principales sistemas del ecosistema digital de la Ciencia Abierta, pudiendo llegar a un equilibrio entre mantener una cuidada identidad digital como investigador, obtener una adecuada visibilidad de su producción de forma transparente para la Comunidad Científica y la Sociedad en general y el tiempo invertido para conseguirlo.

El resto del artículo se organiza como sigue: en la Sección 2 se va a hacer un repaso por los principales indicadores que se deben obtener del ecosistema digital para la Ciencia Abierta; en la Sección 3 se detallará el protocolo para definir la identidad digital de un investigador; por último, la Sección 4 cierra el artículo con las conclusiones de este.

\section{CONTEXTO}

Son muchos los indicadores que se pueden extraer de los diferentes componentes del ecosistema digital de la Ciencia Abierta. Estos se podrían catalogar en cuatro grupos:

1. Indicadores curriculares globales: son aquellos que resumen de una forma global la producción científica de un investigador, como, por ejemplo, su índice h, citas totales recibidas, etc.

2. Indicadores cualitativos por elemento producido: son aquellos que aportan información cualitativa referida a cada aportación (artículo, patente, etc.) del currículo, como, por ejemplo, índice de impacto, cuartil, citas recibidas, etc.

3. Indicadores relacionados con la labor como revisor de artículos científicos: gracias a la incorporación de Publons en el ecosistema digital de la Ciencia Abierta, se puede llevar un registro de la actividad verificada como revisor tanto en revistas científicas como en conferencias académicas.

4. Indicadores relacionados con la visibilidad de un investigador y de su producción científica: son aquellos que dan información de la visibilidad de un investigador y de su producción científica en un determinado medio social, como, por ejemplo, seguidores, visitas a un perfil, descargas de artículos a texto completo, etc.
Cada investigador deberá seleccionar aquellos indicadores que considere más adecuados para la configuración de su identidad digital, congruentemente con la naturaleza de los perfiles digitales que haya configurados en los diferentes componentes del ecosistema digital.

De forma más exhaustiva se van a listar los indicadores curriculares globales (Tabla 1) y los indicadores cualitativos por elemento producido (Tabla 2), por ser estos los más solicitados en los currículos oficiales.

Tabla 1. Principales indicadores curriculares globales

\begin{tabular}{|l|}
\hline \multicolumn{1}{|c|}{ Indicador } \\
\hline $\begin{array}{l}\text { Índice } \mathrm{h} \text { del investigador en cada base de datos de referencia a una } \\
\text { fecha determinada }\end{array}$ \\
\hline $\begin{array}{l}\text { Número de citas totales del investigador en cada base de datos de } \\
\text { referencia a una fecha determinada }\end{array}$ \\
\hline $\begin{array}{l}\text { Promedio de citas del investigador en cada base de datos de } \\
\text { referencia en un período de tiempo (normalmente } 5 \text { últimos años) } \\
\text { a una fecha determinada }\end{array}$ \\
\hline $\begin{array}{l}\text { Cuántos artículos se tienen publicados dentro de una determinada } \\
\text { base de datos de referencia a una fecha determinada }\end{array}$ \\
\hline $\begin{array}{l}\text { Cuántos artículos en revista se tienen publicados dentro de una } \\
\text { determinada base de datos de referencia a una fecha determinada }\end{array}$ \\
\hline $\begin{array}{l}\text { Cuántos artículos en revista se tienen en cada cuartil dentro de una } \\
\text { determinada base de datos de referencia a una fecha determinada }\end{array}$ \\
\hline
\end{tabular}

Tabla 2. Principales indicadores cualitativos por elemento producido

\begin{tabular}{|l|}
\hline \multicolumn{1}{|c|}{ Indicador } \\
\hline $\begin{array}{l}\text { Principales bases de datos en las que se encuentre indexado el } \\
\text { artículo, la patente, etc. a una fecha determinada }\end{array}$ \\
\hline Factor de impacto en cada base de datos en la que se encuentre \\
indexado el artículo en el año de publicación (o del último año de \\
publicación del índice si el correspondiente a su año de \\
publicación todavía no está disponible) a una fecha determinada
\end{tabular}

Para obtener el índice h, las citas totales y el promedio de las citas en los últimos $n$ años, las únicas fuentes que se consideran adecuadas, a la fecha, para incorporarlas a un currículo oficial son WoS, Scopus y Google Scholar (GS). El valor de estos indicadores en las diferentes bases de datos no va a coincidir porque el número de ítems sobre los que se van a calcular no son los mismos. Por ello, cada pareja indicador-valor se debe acompañar de la fuente y de la fecha de consulta. Idealmente, se deberían aportar los indicadores y sus valores de todas las bases de datos en las que el investigador tenga presencia porque de esta forma estará dando una mayor información para su evaluación, debido a que cada base de datos aporta un valor diferente, por ejemplo, WoS y Scopus son más restrictivas y sus indicadores se calcula solo en función de las fuentes que se hayan indexadas en ellas, mientras que GS tiene una cobertura académica universal, incluyendo literatura gris (FerrerasFernández, García-Peñalvo, \& Merlo-Vega, 2015) y no discriminando por idioma o por rama de conocimiento.

Se debe tener en cuenta que solo JCR, dentro de WoS, y Scopus organizan sus contenidos en categorías que se ordenan en función de un determinado factor de impacto dando lugar a 
los cuartiles. En otras bases de datos solo se podrá reportar que la fuente se encuentra indexada, pero no se podrá hacer referencia a cuartil o a índice de impacto.

En el caso de Scopus existen dos factores de impacto de referencia que toman a esta base de datos como fuente. Además, ambos índices se calculan de forma diferente por lo que una revista indexada en Scopus puede aparecer en diferentes cuartiles en función del índice que se tome como referencia. Estos dos índices son el SJR (Scimago Journal Rank), accesible en http://www.scimagojr.com, y el CiteScore, accesible en https://www.scopus.com/sources.

Incluir marcas de tiempo que complementen los valores de los indicadores, que se utilizan en los currículos oficiales o para conformar la identidad digital de un investigador, es algo fundamental porque no se debe olvidar que los valores de estos indicadores se calculan de forma automática por los sistemas informáticos que sustentan este ecosistema digital, es decir, son valores que son susceptibles de cambiar en el tiempo que transcurre desde que el investigador los captura y el evaluador los consulta, con el añadido de que esta variación no siempre se produce de forma positiva para el investigador. Por ello, además de indicar la marca de tiempo, sería recomendable que el investigador recopilase algún tipo de evidencia, como una captura del panel de control o dashboard en la que se puedan apreciar los valores de los indicadores. Esto es especialmente sensible cuando el indicador se está aportando en el contexto de alguna convocatoria oficial en el que el currículo del investigador va a ser evaluado formalmente.

\section{DESCRIPCIÓN}

Son múltiples los componentes y servicios que configuran el ecosistema digital para la Ciencia Abierta. Cada uno de ellos aporta alguna ventaja potencial al investigador, pero también requieren de un importante esfuerzo por su parte para mantenerlos actualizados, curados y obtener así el beneficio potencial que se le presume.

Buscando un equilibrio entre coste (medido en esfuerzo de mantenimiento y curación) y beneficio (medido en obtener los indicadores de evaluación de la investigación y visibilidad potencial de la producción y del investigador), se ha definido un protocolo, que ha ido modificándose en función de la propia evolución del ecosistema digital para la Ciencia Abierta, y del cual se presenta en este artículo su versión más actual compuesto por los siguientes nueve pasos.

\section{A. Elección del nombre de investigador}

La forma en la que un investigador firma sus contribuciones científicas debe representar un identificador alfanumérico que sea consistente para facilitar su identificación en el ecosistema digital, la asignación de sus publicaciones y el cómputo de las citas recibidas por su producción científica.

Este identificador elegido como nombre de autor posibilita tanto una identificación consistente como investigador y la recogida de datos a un nivel más granular, como la agregación de tales datos generando agrupaciones en torno a un determinado investigador, una organización o una determinada fuente de financiación (Gunn, 2013).

El nombre con el que se firmen los trabajos académicos debe ser único y consistente, para ayudar a diferenciar a investigadores con coincidencia parcial de nombres y apellidos, por tanto, es muy recomendable hacer la elección de dicho nombre en un momento temprano de la carrera académica $\mathrm{y}$ mantenerlo una vez elegido.

En el caso de los investigadores no anglosajones puede haber problemas, por ejemplo, los investigadores latinos tienen más de un apellido, al contrario de los anglosajones, y pueden contener caracteres propios como acentos o eñes.

Para la elección del nombre académico de los investigadores latinos se dan las siguientes recomendaciones:

- Apellidos: Si se firma con dos apellidos, deberían unirse con un guion; No se deben abreviar; Se pueden conservar los caracteres propios del idioma (acentos, $\tilde{n}$, etc.).

- Nombre: No usar solo la inicial; Se pueden conservar los caracteres propios del idioma (acentos, ñ, etc.); En los nombres compuestos hay recomendaciones de unirlos con guion (pero no es una práctica extendida ni obligada), además si se quiere abreviar alguno de los nombres usar inicial y punto en lugar de algunas abreviaturas establecidas, por ejemplo, para abreviar María, debería utilizarse M. en lugar de $\mathrm{M}^{\mathrm{a}}$.

\section{B. Creación y mantenimiento de un perfil en ORCID}

ORCID (Open Researcher and Contributor ID http://orcid.org) es una organización que intenta asegurar que todos los trabajos científicos puedan ser atribuidos adecuadamente a sus autores. Para ello ofrece a sus miembros un identificador digital persistente de 16 dígitos, denominados identificadores ORCID, de forma que se mantiene un registro central de sus miembros.

El identificador ORCID sirve principalmente para identificar a un mismo investigador con varias firmas diferentes $y$ distinguir investigadores con nombres de autor que pueden entrar en ambigüedad. Además, sirve como nexo de información e interoperabilidad entre diferentes componentes del ecosistema digital de Ciencia Abierta.

Actualmente, contar con un identificador ORCID es algo que se puede considerar casi como obligatorio y es un metadato cada vez más requerido para autores, editores y revisores en las plataformas de revistas digitales. Por otra parte, no es una red social, ni un anotador de referencias bibliográficas, tampoco es una fuente de indicadores y métricas para la evaluación del investigador.

\section{Creación, curación y mantenimiento de un perfil WoS (ResearcherID / Publons)}

ResearcherID (http://www.researcherid.com) es un identificador único creado inicialmente por Thomson Reuters y ahora propiedad de Clarivate Analytics.

Desde el 15 de abril de 2019 el entorno clásico de ResearcherID dejó de estar operativo y se integró con Publons (https://publons.com). Los usuarios que contasen con un perfil público en ResearcherID y un perfil en Publons habrán quedado fusionados automáticamente y ambas URL llevan al nuevo entorno. El nuevo entorno busca mejorar la experiencia de usuario de ResearcherID porque su interfaz había quedado obsoleta.

Este identificador es voluntario y lo genera el propio investigador. La integración de ResearcherID con ORCID es totalmente recomendable, tanto para el login en ResearcherID / 
Publons como para el intercambio de información entre ambos perfiles, intercambio que se puede hacer bidireccionalmente.

En el perfil de ResearcherID / Publons un investigador puede obtener los indicadores índice $\mathrm{h}$, número total de citas $\mathrm{y}$ promedio de citas en los n últimos años, donde los valores de estas métricas se corresponden con la base de datos de WoS.

\section{Identificación y curación del perfil en Scopus}

A diferencia del identificador ResearcherID / Publons, en la base de datos de Scopus (https://www.scopus.com/) no se puede crear un identificador voluntariamente como fruto de una decisión del investigador, sino que es el sistema el que identifica a los autores de todos los artículos que se indexan en su base de datos. Cada investigador identificado automáticamente mediante una cadena de firma tiene un identificador en la base de datos Scopus, AuthorID. Esto implica que un autor que ha utilizado diferentes maneras de firmar artículos indexados en Scopus tendrá tantos AuthorID como firmas diferentes. Por tanto, hay una labor de identificación, de curación y de fusión de identificadores para poder recuperar los indicadores de un investigador en esta base de datos: índice h, número total de citas y promedio de citas en los n últimos años.

El intercambio con ORCID se realiza de forma unidireccional desde Scopus hacia ORCID, pero no se pueden importar registros desde ORCID a Scopus.

\section{E. Creación y curación de un perfil en Google Scholar}

Google Scholar (en adelante GS) (https://scholar.google.es/) es la mayor base de datos de publicaciones científicas que existe, con un tamaño estimado de 389 millones de registros (Gusenbauer, 2019).

Es uno de los principales perfiles de un investigador de cara a potenciar su visibilidad y su reputación científica, cuando se configura el perfil para que sea público. Estos perfiles públicos permiten compararse entre colegas y entre instituciones, además de acceder a información muy relevante de cara al citado de trabajos académicos.

Los indicadores que se pueden obtener a partir de GS son: índice $\mathrm{h}$, índice $\mathrm{i} 10$, número total de citas y promedio de citas en los n últimos años.

Las métricas que calcula dan una idea de la presencia global que tiene el investigador en la comunidad académica porque no restringe el campo de indexación como lo hace WoS o Scopus.

La mayoría de revistas científicas y de las editoriales que publican en formato digital son accedidas e indexadas por GS, Pero de todas formas la mejor manera de asegurarse de que GS indexe un trabajo es subirlo a un repositorio institucional (Ferreras-Fernández, 2016, 2018; Ferreras-Fernández, MerloVega, \& García-Peñalvo, 2013a, 2013b; García-Peñalvo, 2017b; González-Pérez, Glasserman Morales, RamírezMontoya, \& García-Peñalvo, 2017), respetando los derechos de explotación.

Un perfil público en GS es una herramienta recomendable porque está internacionalmente aceptada; es gratuita; proporciona transparencia; es útil a su autor/institución; y potencia la presencia y visibilidad del investigador con independencia de la rama del conocimiento (lo que beneficia a Ciencias Sociales y Humanidades) y del idioma de publicación (lo que beneficia a autores que publiquen en otros idiomas diferentes del inglés).

No obstante, GS no está libre de errores, lo que obliga al investigador a un importante esfuerzo de curación, el cual se verá reducido si se configura para que no se actualice automáticamente y sea el investigador quien controle la inclusión de nuevos registros al perfil personal, evitando así la asignación de trabajos académicos que no son de la autoría del investigador.

Los principales errores a los que se debe enfrentar un investigador a la hora de curar su perfil de GS son:

- Inclusión de artículos que no son obra del investigador.

- Borrado de artículos que sí pertenecen al investigador.

- Registros duplicados.

- Fusión de documentos que no son el mismo.

Dada la importancia que tiene este perfil para los investigadores, se va a dar una serie de recomendaciones para mejorar la visibilidad del investigador y facilitar, dentro de lo posible, su curación:

- Crear el perfil público.

- Incluir el ORCID después del nombre del investigador.

- Poner la dirección de la página pública de ORCID como página principal en el perfil de GS.

- Usar el correo institucional.

- Poner el nombre oficial de la institución separado por puntos.

- Escribir las palabras clave en inglés.

- No activar las actualizaciones automáticas.

- Mantener el perfil "limpio", al menos los h artículos que otorgan el índice h, recomendable llegar a los que otorgan el índice i10.

- Evitar duplicidades.

- Cuidar la meta-información de las entradas.

\section{F. Creación y mantenimiento de un perfil en ResearchGate}

ResearchGate (https://www.researchgate.net) es la red social de investigadores más importante que existe en la actualidad, con un tamaño estimado de más de 100.000.000 de documentos.

Este componente del ecosistema digital de Ciencia Abierta no está orientado a conseguir indicadores para la evaluación del investigador, aunque podría proporcionar los clásicos (índice h, número de citas totales y promedio de citas en los últimos $\mathrm{n}$ años), pero su validez no se considera adecuada porque su cobertura (a diferencia de WoS, Scopus o GS) no es completa. Por tanto, su misión es dar visibilidad a la producción científica de un investigador y a establecer una red de contactos y de interacción.

Hace posible compartir contenidos científicos, tanto publicaciones como conjuntos de datos. Permite consultas abiertas a la comunidad de investigadores, a la vez que facilita recomendar habilidades y competencias de otros investigadores. ResearchGate tiene un sistema de avisos orientados a que el investigador tenga conocimiento de lo que sucede en su red social, especialmente en lo tocante a su producción. Para asociar la producción científica a un investigador, el sistema combina el descubrimiento de contribuciones de forma automática con las acciones de incorporación de trabajos de forma manual por parte del autor. 
Además de los clásicos indicadores ya comentados, ResearchGate aporta otros dos que tampoco se recomienda que se usen para su inclusión en currículos oficiales y tienen más un sentido de gamificación dentro de la red social. Estos son el ResearchGate Score, que es una forma de medir la reputación científica del investigador y se calcula en base a las publicaciones en el perfil personal y cómo otros investigadores interaccionan con el contenido en la red social ResearchGate, y el Research Interest, que es una indicador que se incorpora en febrero de 2019 y mide el interés de la comunidad científica en la producción global (o en cada uno de los artículos) de un autor, es la suma del Research Interest de cada ítem añadido al perfil, donde cada ítem tiene su propio valor basado en las citas, recomendaciones y lecturas que haya recibido.

\section{G. Creación, curación y mantenimiento de un perfil como revisor en WoS (ResearcherID / Publons)}

Publons (https://publons.com) nace con el objetivo de registrar la actividad de revisión y edición de artículos científicos, así como alentar a los investigadores a publicar sus experiencias online como árbitros. De hecho, el nombre Publon significa la unidad fundamental de investigación científica que justifica ser publicada.

La revisión por pares es un trabajo especializado y valioso, que se pierde después de la publicación del artículo, desperdiciando así, ideas, sugerencias y comentarios. La finalidad de Publons es reunir y almacenar este contenido, promoviendo discusiones. Además, trata de reconocer $\mathrm{y}$ convertir la revisión por pares en una medida de producción científica.

Como ya se ha comentado previamente, en abril de 2019 se integra con ResearcherID de WoS, pero sigue existiendo la funcionalidad de registro de la actividad de revisión, por ello se ha decido separar ambas prestaciones en pasos distintos del protocolo, aunque se integran en el mismo componente del ecosistema digital de Ciencia Abierta.

Todos los registros de revisión/edición que se incorporan al perfil del investigador en Publons se tienen que verificar mediante los correos electrónicos que recibe el revisor/editor después de finalizar una revisión o tomar la decisión de aceptación/rechazo de un artículo científico. Esto permite generar un informe de evidencias con las revisiones realizadas para aportarlo a cualquier proceso de evaluación del investigador.

\section{H. Registro de la identidad digital en un formato de fácil consulta y actualización}

Dado que los indicadores y métricas procedentes de los diferentes perfiles se pueden usar en diversos procesos de evaluación y acreditación, su registro en un documento, con una estructura fácil de consultar y actualizar, que refleje la marca de tiempo de los datos obtenidos, es una tarea necesaria para hacer explícita la identidad digital creada como investigadores.

De cada uno de los perfiles que un investigador mantenga, se debe seleccionar sus principales indicadores para documentar sus valores.

\section{Divulgación (propagación) de la actividad}

La divulgación a la sociedad (García-Peñalvo, 2016) es un cometido de los investigadores y una tarea complementaria y necesaria al proceso de publicación para dar a conocer la investigación realizada. Esto se puede hacer mediante múltiples canales (redes sociales, blogs, perfiles especializados, etc.).

El objetivo es conseguir mayor visibilidad y reconocimiento (Crue \& FECYT, 2018), pero a cambio la inversión en tiempo para llevar a cabo esta tarea es muy elevada. Es una decisión importante la elección del canal o canales de divulgación, equilibrando el esfuerzo de mantenimiento del canal y el retorno potencial.

De todos los canales posibles, Twitter es una red generalista de microblogging que puede resultar muy adecuada para la divulgación científica debido a las características del mensaje en esta red social: intuitivo, rápido, preciso, eficaz en el mensaje y que enlaza con las altmétricas (Galligan \& Dyas-Correia, 2013). Esto permite que el investigador pueda definir diferentes estrategias de comunicación científica gestionando diversos estilos de tuits y el tiempo invertido en ello.

Independientemente del estilo de comunicación que se utilice, hay una serie de recomendaciones que se pueden seguir para la divulgación científica en Twitter:

- Conjugar un rol de transmisor de información con un rol de participación en discusiones científicas.

- Combinar tuits en los que se da información de un artículo con tuits en los que se da información (no copy/paste) relevante sobre los contenidos de dicho artículo.

- Aportar imágenes y gráficos de la investigación.

- Se debe aportar una URL o DOI del artículo.

- Se pueden aportar informaciones relativas a eventos, grupo, etc.

- Se debe tener una orientación más de información que de opinión.

- Mantener una presencia equilibrada.

\section{CONCLUSIONES}

La Ciencia del siglo XXI está ligada a la Ciencia Abierta a través del ecosistema digital en el que la identidad digital de un investigador es el reflejo de sus perfiles digitales en dicho ecosistema.

Los perfiles digitales de los investigadores transmiten transparencia y otorgan visibilidad a su producción científica, lo que potencialmente se reflejará en los indicadores que influyen en la evaluación de la investigación. Por tanto, tener una identidad digital como investigadores es un acto de transparencia y rendición de cuentas con la sociedad. Pero hay que mantenerlos actualizados y en el caso de que se alimenten automáticamente deben curarse para que no se nutran de fuentes incorrectas o repetidas que puedan dar una imagen equivocada del investigador y en un cálculo incorrecto de los indicadores que se emplean para la evaluación de un investigador.

El beneficio potencial de los perfiles digitales para un investigador es muy alto, pero es innegable que también supone un trabajo arduo, pesado, por tanto, se deben elegir solo aquellos perfiles digitales que un investigador sea consciente de que le va a dedicar el tiempo necesario.

Para ayudar a tomar la decisión de qué presencia tener en este ecosistema digital de la Ciencia Abierta, tan complejo y cambiante, se ha definido un protocolo que guíe al investigador y le permita obtener el máximo retorno en base al esfuerzo invertido, porque hay unos perfiles que un investigador del siglo XXI no puede permitirse no tener y no cuidar (WoS, Scopus, ORCID, GS, ResearchGate). 
Para Ciencias Sociales y Humanidades, GS da una mayor visibilidad que otras bases de datos como WoS o Scopus.

Actualmente, uno de los factores que más influyen en la visibilidad y capacidad de ser citado es la presencia en las redes sociales académicas de una manera activa. Las citas, hoy en día, son el indicador que mayor peso específico, directa e indirectamente, tiene en la evaluación de la actividad investigadora.

Para lograr los mejores resultados potenciales en visibilidad, transparencia y retorno, la presencia y el mantenimiento de los perfiles digitales se debe completar con un conjunto de buenas prácticas propias de la Ciencia Abierta como cuidar los metadatos de las publicaciones, publicar en abierto (GarcíaPeñalvo, García de Figuerola, \& Merlo-Vega, 2010; RamírezMontoya, García-Peñalvo, \& McGreal, 2018), combinando la ruta dorada y la ruta verde (Piwowar et al., 2018) y divulgar la producción científica en redes sociales especializadas y generalistas.

\section{AGRADECIMIENTOS}

Los autores agradecen a la Universidad de Zaragoza por su apoyo y financiación en el proyecto de Innovación Docente PRAUZ_18_076.

\section{REFERENCIAS}

Bohannon, J. (2016). Hate journal impact factors? New study gives you one more reason. Retrieved from https://bit.ly/2Z7XwXD

Crue, \& FECYT. (2018). Guía de valoración de la actividad de divulgación científica del personal académico $e$ investigador Retrieved from https://goo.gl/uAHWMe

Crue Universidades Españolas. (2019). Compromisos de las universidades ante la Open Science. Madrid: Crue Universidades Españolas Retrieved from https://goo.gl $/ \mathrm{mRB} 2 \mathrm{zA}$.

DORA. (2013). San Francisco Declaration on Research Assessment. Retrieved from https://sfdora.org/read/

Fecher, B., \& Friesike, S. (2014). Open Science: One Term, Five Schools of Thought. In S. Bartling \& F. S. (Eds.), Opening Science. The Evolving Guide on How the Web is Changing Research, Collaboration and Scholarly (pp. 17-47). Cham: Springer.

Ferreras-Fernández, T. (2016). Visibilidad e impacto de la literatura gris cientifica en repositorios institucionales de acceso abierto. Estudio de caso bibliométrico del repositorio Gredos de la Universidad de Salamanca. (PhD), Universidad de Salamanca, Salamanca, España. Retrieved from https://goo.gl/rrNeEJ

Ferreras-Fernández, T. (2018). Los repositorios institucionales: Evolución y situación actual en España. In J. A. Merlo Vega (Ed.), Ecosistemas del Conocimiento Abierto (pp. 39-84). Salamanca, España: Ediciones Universidad de Salamanca.

Ferreras-Fernández, T., García-Peñalvo, F. J., \& Merlo-Vega, J. A. (2015). Open access repositories as channel of publication scientific grey literature. In G. R. Alves \& M. C. Felgueiras (Eds.), Proceedings of the Third International Conference on Technological Ecosystems for Enhancing Multiculturality (TEEM'15) (Porto, Portugal, October 7-9, 2015) (pp. 419-426). New York, USA: ACM.

Ferreras-Fernández, T., Merlo-Vega, J. A., \& García-Peñalvo, F. J. (2013a). Impact of Scientific Content in Open Access Institutional Repositories. A case study of the Repository Gredos. In F. J. García-Peñalvo (Ed.), Proceedings of the First International Conference on Technological Ecosystems for Enhancing Multiculturality (TEEM'13)
(Salamanca, Spain, November 14-15, 2013) (pp. 357-363). New York, NY, USA: ACM.

Ferreras-Fernández, T., Merlo-Vega, J. A., \& García-Peñalvo, F. J. (2013b). Science 2.0 supported by Open Access Repositories and Open Linked Data. In F. J. García-Peñalvo (Ed.), Proceedings of the First International Conference on Technological Ecosystems for Enhancing Multiculturality (TEEM'13) (Salamanca, Spain, November 14-15, 2013) (pp. 331-332). New York, NY, USA: ACM.

Galligan, F., \& Dyas-Correia, S. (2013). Altmetrics: Rethinking the Way We Measure. Serials Review, 39(1), 56-61. doi:10.1016/j.serrev.2013.01.003

García-Peñalvo, F. J. (2016). La tercera misión. Education in the Knowledge Society, $\quad$ 17(1), 7-18. doi:10.14201/eks2016171718

García-Peñalvo, F. J. (2017a). ¿Cómo construir un perfil digital de investigador en Innovación Educativa? . Paper presented at the IV Congreso Internacional sobre Aprendizaje, Innovación y Competitividad. CINAIC 2017 (4-6 de Octubre de 2017), Zaragoza, España. https://goo.gl/zFpHxu

García-Peñalvo, F. J. (2017b). The Future of Institutional Repositories. Education in the Knowledge Society, 18(4), 7-19. doi:10.14201/eks2017184719

García-Peñalvo, F. J. (2018a). Identidad digital cientifica. Paper presented at the Jornadas CINAIC 2018 - EcoAprendemos, CICEI (Centro de Innovación para la Sociedad de la Información, Universidad de las Palmas de Gran Canaria. https://goo.gl/vw6cHT.

García-Peñalvo, F. J. (2018b). Identidad digital como investigadores. La evidencia y la transparencia de la producción científica. Education in the Knowledge Society, 19(2), 7-28. doi:10.14201/eks2018192728

García-Peñalvo, F. J., García de Figuerola, C., \& Merlo-Vega, J. A. (2010). Open knowledge: Challenges and facts. Online Information Review, 34(4), 520-539. doi:10.1108/14684521011072963

González-Pérez, L. I., Glasserman Morales, L. D., Ramírez-Montoya, M. S., \& García-Peñalvo, F. J. (2017). Repositorios como soportes para diseminar experiencias de innovación educativa. In M. S. Ramírez-Montoya \& J. R. Valenzuela González (Eds.), Innovación Educativa. Investigación, formación, vinculación y visibilidad (pp. 259-272). Madrid, España: Síntesis.

Gunn, W. (2013). Social signals reflect academic impact: What it means when a scholar adds a paper to Mendeley. Information standards quarterly, 25(2), 33-39. doi:10.3789/isqv25no2.2013.06

Gusenbauer, M. (2019). Google Scholar to overshadow them all? Comparing the sizes of 12 academic search engines and bibliographic databases. Scientometrics, 118(1), 177-214. doi:10.1007/s11192-018-2958-5

Hatch, A., \& Curry, S. (2018). Evaluation woes: we're on it, responds DORA. Nature, 559, 32. doi:10.1038/d41586-018-05596-x

Piwowar, H., Priem, J., Larivière, V., Alperin, J., Matthias, L., Norlander, B., . . . Haustein, S. (2018). The State of OA: A large-scale analysis of the prevalence and impact of Open Access articles. PeerJ, 6, e4375.

Ramírez-Montoya, M. S., García-Peñalvo, F. J., \& McGreal, R. (2018). Shared Science and Knowledge. Open Access, Technology and Education. Comunicar, 26(54), 1-5.

Tregoning, J. (2018). How will you judge me if not by impact factor? Nature, 558, 345. doi:10.1038/d41586-018-05467-5

Vicente-Saez, R., \& Martínez-Fuentes, C. (2018). Open Science now: A systematic literature review for an integrated definition. Journal of Business Research, 88, 428-436. doi:10.1016/j.jbusres.2017.12.043 